\title{
Collaborative Cellular-based Location System ${ }^{\star}$
}

\author{
David Navalho, Nuno Preguiça \\ CITI / Dep. de Informática - Faculdade de Ciências e Tecnologia \\ Universidade Nova de Lisboa, \\ Quinta da Torre, 2829-516 Caparica, Portugal
}

\begin{abstract}
A large number of techniques for location estimation relying on the existing wireless radio infrastructures have been proposed in the last few years. Solutions relying on the use of cellular networks can be used almost everywhere, but tend to have low precision. In this paper, we propose the use of information sharing among nearby mobile phones for improving accuracy of cellular-based location. Our information sharing solution was developed in the context of UCLS, a modular location system. This mechanism allows a mobile phone to use additional signal-strength information obtained from nearby devices, that can be connected to different cells of the same or of a different operator. Results show that this additional information helps improving results, with accuracy over $94.8 \%$ when using only 2 mobile phones connected to different cell towers when locating the user at the room-level.
\end{abstract}

\section{Introduction}

In recent years, mobile phones have become smaller, more powerful and less expensive. Additionally, they start including several short-range radio technologies that can be used for building location systems, such as Bluetooth [1] and Wi-Fi $[2,5]$. Additionally, some devices can also provide location relying on the included GPS hardware. These location mechanisms have allowed the development of a large number of location-aware applications and services [8] that are becoming fundamental to users, with GPS location and navigation as the most widely publicized and used application.

Taking advantage of the technologies available in mobile devices, several location systems have been proposed in literature $[1,2,15,13,5]$. An approach that became popular is to determine the location using information obtained from the existing wireless radio infrastructures. This approach has the advantage of being easy to deploy as it requires no additional hardware. To implement this approach, several techniques and radio systems can be used. For example, Radar [2], a pioneer work in this area explored two location techniques using the existing Wi-Fi infrastructure.

Some works have also explored the use of this approach with the existing cellular networks $[3,15]$. In contrast with Wi-Fi networks that are only available

\footnotetext{
* This work was partially supported by FCT/MCTES, project \#PTDC/EIA/76114/2006 and CITI.
} 
in limited areas, cellular networks tend to be available everywhere. This has the potential to allow providing a location-based system with a more complete coverage. However, the accuracy of location estimation using cellular networks tends to be lower than when using Wi-Fi [12].

In this paper, we propose the use of information sharing among nearby mobile devices to improve the accuracy of cellular-based location estimation. Using this approach, a mobile device will use not only the information it obtains locally, but also the information obtained in nearby devices for computing location. The rationale for this idea is that by using a larger number of signal readings, it is usually possible to obtain better results [8].

This is particularly important for cellular-based location. First, unlike previous works that relied on special hardware for retrieving the signal-strength for multiple cells $[13,14]$, our approach allows nearby mobile phones to exchange the signal-strength information for the cell they are connected to, the only information that can be accessed using the existing APIs in common mobile devices. The use of the signal-strength from multiple cells is essential for providing good location results.

Second, even for special hardware without the previous software limitation, it is usually only possible to obtain information for the cells of a single operator [13, 14]. As it has been previously noticed [3], using weak signal strengths tends to make more harm than good. Our approach, when used to exchange information among users connected to different networks allows to gather a larger number of strong signals, thus potentially leading to better results.

Our tests show that by using this approach, it is possible to identify correctly the room a user is in more than $94.8 \%$ of the cases, when using information from only 2 mobile phones connected to different cells. These results are feasible in our network environment because, in a given place, the signal strength for cells of different operators tend to be different. Moreover, the signal strength for the cells of the same operator tend to be different when connected to GSM or UMTS.

These results and our approach have been implemented in the context of the Unified Cooperative Location System (UCLS), a modular location system that supports different location techniques and technologies. The UCLS information sharing mechanism allows a mobile device to exchange location-related information with nearby devices. This mechanism can be explored in several ways, not only the one mentioned earlier. First, it can make available to a device information from technologies that it does not include. As an example, a GPS-less device can access GPS information from a device that includes GPS hardware. This is already common in devices that can access a Bluetooth GPS. Second, it allows a device to obtain additional information that can be used for improving accuracy. For example, by combining the GPS values of two very close devices, it might be possible to reduce the location error.

The remainder of this paper is organized as follows. Section 2 discusses related work. Section 3 presents the Unified Cooperative Location System's architecture, and section 4 presents our experiments. Finally, the conclusions and future work are presented in section 5 . 


\section{Related Work}

The area of location systems has been the target of a lot of several research in the past years, resulting in a myriad of proposed approaches [8]. Some solutions require the use of custom hardware and deployment $[4,11]$. Although these systems can achieve accuracies of a few centimeters, they also impose extra costs for the hardware and its deployment, not to mention the additional equipment a user usually needs to carry if he wishes to take advantage of the system.

Other solutions take advantage of the existing wireless infrastructures. These approaches have become increasingly popular as they represent low-cost solutions that have good accuracy. The proposed solutions vary greatly in the used technologies and algorithms $[1-3,5-7,10,14,15]$.

In [1], the authors present a system that sends advertisements based on the proximity of users. To locate nearby users, they probe for Bluetooth devices and use the signal strength of devices to estimate their position. In [10], the authors propose a positioning approach relying on PowerLine. The proposed approach uses fingerprinting of multiple tones transmitted along the power line to achieve subroom-level localization.

In Radar [2], location is estimated relying on the use of existing Wi-Fi signals. Two approaches have been experimented in this system. The first, based on the use of fingerprinting techniques. In this approach, it is necessary to start by mapping the target area with wireless signal fingerprints. After this mapping phase, the location of a user is estimated considering the stored fingerprints that are the closest match to the observed one. The second approach, executes triangulation algorithms relying on signal propagation models to compute the distance of the user to each base station. In this work, the authors observed that the first solution worked better and it has a very good accuracy.

In SkyLoc [15], the authors present a fingerprinting-based location system that relies on GSM signals. The system works only with a specific phone able of providing to the applications the signal strengths of multiple GSM towers. Other works on GSM location also tend to use specific phones [3,14]. These works served as inspiration to UCLS. In our work we use a similar fingerprintingbased approach, but instead of relying on a specific mobile phone, we use our information exchanging mechanism to obtain information from multiple cellular towers. This allows our solution to work with common mobile phones, which only provide application information from a single cellular tower.

Several systems have used multiple technologies for providing location [12,7]. In our system, location estimation can also rely on all the available technologies on the mobile phone. Additionally, by obtaining information from nearby devices it can use not only the technologies in the mobile devices but also those in nearby devices. This approach can help in improving accuracy and reduce energy consumption.

The Calibree [16] system also uses information sharing among mobile users. The system combines the distances measured among a set of mobile phones with the location of a small number of GPS-equipped phones to estimate the absolute location of all devices. In our approach, we can combine information from 
GPS-enabled mobile phones to automatically create the mapping information necessary for cellular-based location.

\section{System Architecture}

The Unified Cooperative Location System (UCLS) is a modular location system that includes an information exchanging mechanism. The modular design allows it to adapt to the existing hardware in the target mobile phone, by using different algorithms and associated location information. Depending on the used algorithms and data, the system can provide symbolic, relative and absolute positioning estimation. This estimation can be performed using GPS-obtained information or using solutions that rely on any wireless signals, including Wi-Fi, GSM and UMTS.

The UCLS is structured in four Modules and a collection of Sniffers, as depicted in figure 1. The system runs on commodity mobile phones and was implemented mostly in Java 2 Micro Edition. The only exception are the Sniffer components, which were implemented in Python for S60 (pys60). This was necessary to allow the acquisition of wireless information data, such as signal strength, which cannot be obtained in Java 2 ME.

The system provides a low-cost solution for location, allowing to use the existing technologies on the mobile phone for providing location estimation with good accuracy. The information exchanging mechanism included in the system also contributed to these goals. First, it allows devices without some hardware, such as GPS, to still obtain this type of information from nearby devices. Second, by obtaining additional readings for technologies the device includes, it allows to improve accuracy in some cases - in section 4 we describe our experiments with GSM and UMTS-based location. The client based solution for location estimation implemented in UCLS guarantees privacy to users. We believe our information exchange mechanism only hampers this goal by little. First, exchanged information is anonymous. Second, as Bluetooth only works at shortrange, the users that exchange information are already in very close range. If this is considered a problem, some additional solution could be integrated in our system, such as the one proposed in [17].

\subsection{Location API Module}

This module serves the simple purpose of delivering an API for a developer to use a location system on his application. The API consists of several types of operations: configuration, data manipulation, estimation.

The configuration operations allow applications to define several options of the system: the technologies to be used (GPS, cellular, Wi-Fi, Bluetooth); the algorithms to be used (fingerprinting, averaging); and whether to use the information sharing mechanism or not.

The data manipulation operations include the necessary methods to gather location information for fingerprinting map building. When GPS exists, the system allows the mapping process to run in background automatically. When no 
GPS lock is available, the user needs to add additional information to the fingerprinting information. When obtaining information from multiple devices, the system supports synchronized information gathering, thus allowing to obtain information from multiple devices at the same time. Additionally, the data manipulation operations include support for storing the needed location-related data in the local device or in an external data storage.

Finally, the module provides an operation for location estimation. This operation gathers the surrounding wireless information, including from nearby devices if such option is active, and infers the current position using the active algorithms present in the Location module and the stored data present in the Database Module.

\subsection{Location Module}

The Location Module is in charge of controlling the other Modules of the system. It acts as the coordinator of the system, obtaining wireless location information from the Communications Module, for map building or location estimation. This module also uses the Database Module to store or retrieve the necessary information for executed operations.

Location estimation algorithms are added to the Location Module, which has the necessary capabilities to process them. In our current prototype, we support two existing location algorithms. In this paper, due to lack of space, we only discuss our fingerprinting-based location algorithm (that is a client-based solution similar to others previously proposed - e.g. RADAR [2], SkyLoc [15]).

Fingerprinting Fingerprinting is a simple location estimation method, composed of two phases: an information gathering phase for map construction, and a location estimation phase which uses the previously built map and a recently acquired reading in order to estimate the current location.

The mapping phase consists in collecting, for a set of locations, the signal strength of detected wireless technologies (e.g.: Wi-Fi, GSM, Bluetooth). For each location, the system stores the wireless signal fingerprint and the location information. The stored location information can be acquired with a GPS reader, or manually introduced as a symbolic or relative location. Several solutions have been proposed for improving this process [9], which could be used in our system.

After the intended area for location estimation is fully mapped, a user can use the gathered information for the second phase. The user first acquires a wireless information reading, searching the stored mapping information for the closest match, thus inferring his current position. This is possible thanks to the fact that wireless communications signal strengths do not vary significantly in a given location over time (temporal consistency) while at the same time, they vary significantly from location to location (spatial variability) [8].

Several definitions for closest match have been experimented in other location systems [8]. In our current prototype we are using the Manhattan distance, in which the distance, $d(u, v)$, between two fingerprints vectors $v$ and $u$, is computed 


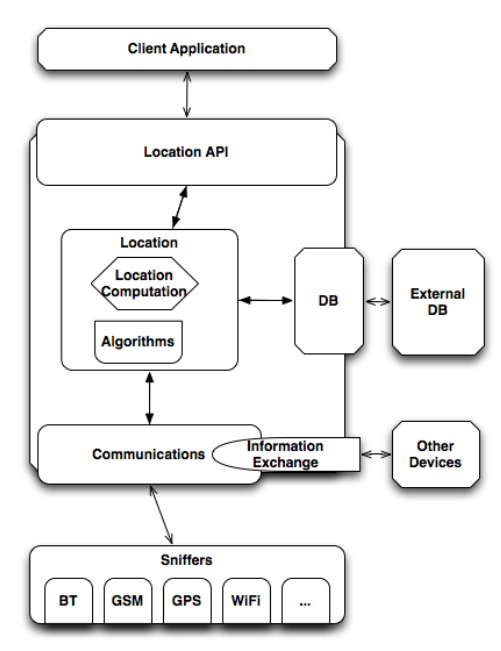

Fig. 1. UCLS Architecture

as follows: $d(v, u)=\sum_{i}\left|v_{i}-u_{i}\right|$. In the vectors, each position corresponds to a different base station/tower.

Some additional parameters were added to the algorithm, in order to address the situations where the observed base stations/towers are different: a configurable handicap value can be added for each unmatched base station/tower; and for multiple results with the same Manhattan value, the one with the most matching elements is chosen as the best.

\subsection{Communications Module}

The Communications Module is responsible for gathering wireless information and reporting the results to the Location Module. This module communicates with the available Sniffers to obtain this information.

Additionally, a set of bluetooth primitives are also available, currently using the Java 2 Micro Edition package for bluetooth (JSR82), when available. These primitives are mainly used to search for devices running the information exchange service, though they are also used by the Database Module to communicate with another computer for data sharing or acquisition (when Wi-Fi is unavailable).

Sniffers Since the J2ME environment does not provide the needed information for location estimation, small modules were implemented using the pys60 language, for each available technology. The modules and communication were made as simple and modular as possible, allowing additional Sniffers to be added without the need to modify the Communications Module. We chose Python for 
S60 since it provides the necessary tools for obtaining the required information location, and a very simple programming environment.

Each Sniffer acts as a simple TCP server, waiting to receive an activation message. While a technology like GSM does not actually need activation, since the data is always available, one like GPS uses energy whenever it is active. In order to provide energy saving primitives, the ability to activate and deactivate the Sniffers is necessary. Therefore, a deactivation message is also available, so that a user or the system can deactivate a technology when it is deemed not necessary. Other then that, the TCP server only receives wireless information reading requests, replying back with the gathered information.

Information Exchange The Information Exchange mechanism present in the Communications Module is responsible for exchanging location-related information with nearby devices. This module exports a Bluetooth service that allows other devices to obtain information gathered locally. We have decided to use only bluetooth for communication due to its short range and lower energy consumption, since we only want to contact devices that are close enough to provide useful information.

When an available device for sharing is detected, any kind of location information can be exchanged. For example, a device with less capabilities can obtain the GPS location information from a nearby device with such functionality. Additionally, this method allows a device to obtain the raw signal strength readings from the nearby devices, and use it for both map building and location estimation. Depending on the technology and distance between devices, this information can be used to average the error of individual readings, as well as allowing to improve location accuracy.

There are some points to take into account when implementing an information exchange service for location. The first is how useful the information can be used to improve accuracy. Bluetooth can reach only a few meters at most of range, thus users must be very close to each other when sharing location information. Although this adds some error, one can still obtain improvements when the error of the location technique used is much higher than a couple of meters, like when using GSM. Unfortunately, the Symbian implementation for bluetooth does not provide the signal strength information of a bluetooth communication, which could eventually help to estimate the relative distances between users. In the future, we intend to extract this information, as in [16], and use it to lower the estimation error while sharing location information.

When sharing information, an important aspect is whether the users are moving or not. If users are moving, it is important to have the readings at the same time (or as close as possible). To this end, the service provides a reading timed request, which basically performs a synchronized reading.

\subsection{Database Module}

The Database Module allows other modules to store and retrieve location-related information. When using fingerprinting-based solutions, this module basically 


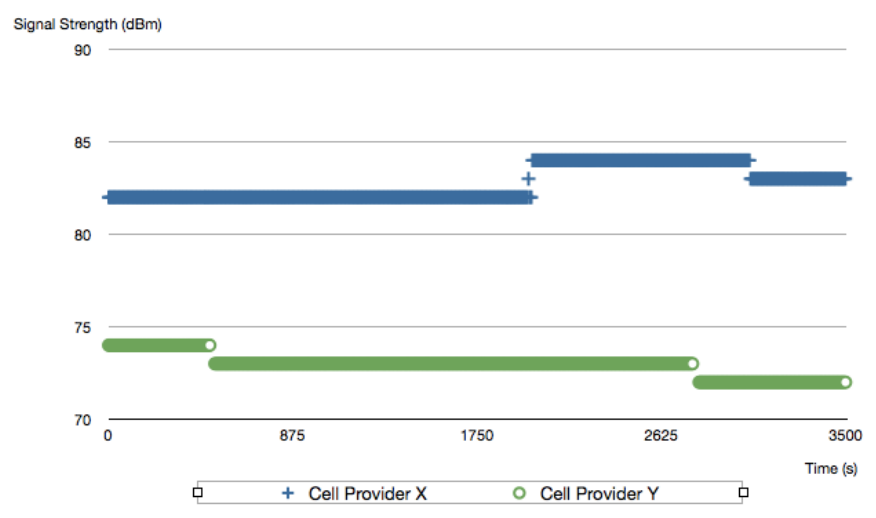

Fig. 2. GSM Signal variation over time

stores fingerprint and associated location information. This module can use information stored only locally or access information stored in an external server. In this late case, the database module can cache information from the server when necessary.

Using a server to store location-based information allows for data sharing among users of the system. This greatly simplifies the adoption of this type of system, by cooperatively adding the needed information to the system - this approach is used in several systems [7].

\section{Experiments}

In this section we present results obtained using UCLS with fingerprinting location algorithms.

We start by presenting some results concerning signal stability observed over time. This information is important as location techniques based in signal strength, and in particular fingerprinting solutions, assume that the signal remains (almost) the same over time. If this is not correct, location cannot be estimated correctly. To evaluate the stability of the GSM in a given location, we periodically measured and recorded the signal strength over a long period of time for a mobile phone in the same location. Figure 2 presents the results obtained for two different mobile phones connected to two different cells. These results show that the GSM signal strength varies very little over time, thus presenting the stability property necessary for location estimation.

For our work, we were particularly interested in evaluating if the information sharing mechanism could allow to build a practical solution for cellular-based location in common mobile phones. To this end, we have measured the accuracy of symbolic location estimation with cellular fingerprints gathered at distinct days in the FCT-UNL campus (figure 3). Each cellular fingerprint contains up to 6 signal strength values - these values were obtained using 6 different mobile 


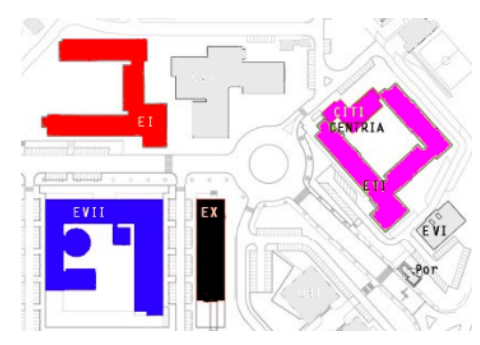

Fig. 3. FCT-UNL campus

phones in two different days; two phones were connected to each of the three operators in the area; for each operator one phone was connected to a GSM tower and the other to a UMTS tower. These cellular fingerprints were obtained using the synchronized shared readings implemented in UCLS.

Based on this data, we performed our accuracy evaluation off-line. Our testing algorithm works as follows. In each iteration, a random shared reading is selected. Then, we use the location estimation algorithm implemented in UCLS to estimate the location of the selected reading, without considering it (i.e., the shared reading that is removed from the database during the iteration). For each selected shared reading, we perform up to six tests. In the first test, we consider only the signal strength of one random tower. In the second test, we use the values of two random towers, and so on. All results presented were achieved by repeating this process 10.000 times.

\subsection{Locating large areas}

Our first test tried to evaluate if it was possible to distinguish the location of users among different building in the campus. To this end, a total of 450 shared readings (or fingerprints) were obtained in four buildings. Each fingerprint contained signal strengths for at least four identified cells (sometimes, a reading from a mobile phone failed because the signal was too weak or some communication error occurred). A total of 1958 individual readings were obtained, referring to 21 distinct cells.

Table 1 presents the accuracy of cellular-based location, when using the granularity of the building. The results obtained are all very good, even when using a single cell, with an accuracy of $86.7 \%$. These good results are explained by several factors. First, for some cells, all the readings for that cell are in the same building. In this case, whenever such a value was selected, the result was always correct. Second, for cells that are observed in different buildings, values tend to be different in different buildings, with one major exception in readings in building EVII (blue) and EX (black).

The results also show an improvement when using the signal strength from different cells - e.g. with only two cells, it was possible to locate the user with an accuracy of $98 \%$. This shows that by using additional information it was possible to minimize the error. 
Table 1. Location accuracy using cells - building

\begin{tabular}{cc}
\hline Number of Cells \% & Building \\
\hline 1 & 86.7 \\
2 & 98.0 \\
3 & 99.1 \\
4 & 99.8 \\
5 & 100.0 \\
6 & 100.0 \\
\hline
\end{tabular}

Table 2. Location accuracy using cells inside a building

\begin{tabular}{|c|c|c|c|}
\hline \multicolumn{4}{|c|}{ Number of Cells \% Room \% Floor \% Wing } \\
\hline 1 & 73.2 & 85.3 & 86.6 \\
\hline 2 & 94.9 & 96.8 & 97.2 \\
\hline 3 & 98.5 & 98.9 & 98.7 \\
\hline 4 & 98.9 & 99.1 & 99.5 \\
\hline 5 & 99.5 & 99.7 & 100.0 \\
\hline 6 & 100.0 & 100.0 & 100.0 \\
\hline
\end{tabular}

\subsection{Locating small areas}

Next, we have evaluated the accuracy of the system with smaller areas: wings, floors and rooms. To this end, we used a single building: EII - the pink building on figure 3 . This building has 3 floors in one wing, and two floors in the other 3 wings. We have obtained additional fingerprints with symbolic information including wing, floor and room. A total of 380 shared readings (fingerprints) were obtained, consisting of 1948 unique signal strengths from 23 unique cell ids.

Table 2 presents the accuracy of location estimation for the different symbolic areas. As expected, the accuracy of room estimation is smaller than that of wing estimation. Even though, accuracy is very good for all areas and improves with the size of the fingerprint used in the search. Even for room-granularity, accuracy is $94.9 \%$ using information from two mobile phones and $98.5 \%$ using information from three mobile phones.

For a final phase of testings, we made two additional adjustments. First, we merged the data obtained when mapping the campus, with the data gathered to map the wings, floors and rooms of the building, in order to obtain a more realistic view of a bigger database. The second adjustment, was to simulate an information exchange between nearby devices that were not in the same position. To this end, our tests started by randomly selecting a room. In this room, instead of using a single fingerprint, we select up to 6 random fingerprints. When testing the location, we now use from 1 to 6 signal strengths from different fingerprints.

Table 3 presents the accuracy results for location estimation at the roomlevel. The second column contains the results obtained by simply merging the database, while the third column contains the results obtained when considering distanced readings. Although the accuracy drops when considering randomly 
Table 3. Location accuracy using cells, for room level, using merged databases

\begin{tabular}{ccc}
\hline Number of Cells \% & Merged \% & Merged and exchanged \\
\hline 1 & 73.1 & 68.0 \\
2 & 94.8 & 86.4 \\
3 & 97.8 & 87.4 \\
4 & 98.8 & 91.0 \\
5 & 99.4 & 91.2 \\
6 & 99.5 & 95.8 \\
\hline
\end{tabular}

distanced readings, the results are still very good - with an accuracy over $86 \%$ when using information from only two cells.

The good results obtained in our experiments suggest that the information sharing mechanism can be used to build a practical cellular-based location system.

\section{Conclusions and future work}

In this paper, we have presented UCLS, a modular system that provides location based on any wireless technology available to a mobile device, with the ability to use any technology of the mobile device in the process of location estimation. Unlike previous systems, UCLS includes an information sharing mechanism that allows nearby devices to share location-related information. This mechanism has two advantages. First, it allows a device to obtain information regarding technologies it does not include. Second, it allows to obtain additional readings, with the potential to improve accuracy and reduce energy consumption.

This mechanism is specially interesting for cellular-based location, as it allows to obtain enough information for determining location with good accuracy. Our tests show that by using this approach, it is possible to identify correctly the room a user is in more than $94.8 \%$ of the cases, when using information from only 2 mobile phones connected to different cells. These results improve even more when using information from additional phones.

Unlike previous solutions that only work on specific hardware, UCLS provides a practical solution that works with common mobile phones.

In the near future, we intend to further improve the algorithm we use in UCLS. One aspect that we would like to include in our algorithms would be to use the estimation of distance between devices when sharing information. Other aspect that we would like to study was the implementation of algorithms that use signal propagation modeling with triangulation.

\section{References}

1. L. Aalto, N. Göthlin, J. Korhonen, and T. Ojala. Bluetooth and wap push based location-aware mobile advertising system. In MobiSys '04: Proceedings of the 2nd international conference on Mobile systems, applications, and services, pages 4958, New York, NY, USA, 2004. ACM. 
2. P. Bahl and V. N. Padmanabhan. Radar: an in-building rf-based user location and tracking system. In INFOCOM 2000. Nineteenth Annual Joint Conference of the IEEE Computer and Communications Societies. Proceedings. IEEE, volume 2, pages 775-784 vol.2, 2000.

3. M. Chen, T. Sohn, D. Chmelev, D. Haehnel, J. Hightower, J. Hughes, A. Lamarca, F. Potter, I. Smith, and A. Varshavsky. Practical metropolitan-scale positioning for gsm phones. In UbiComp 2006: Ubiquitous Computing, pages 225-242. 2006.

4. A. Harter, A. Hopper, P. Steggles, A. Ward, and P. Webster. The anatomy of a context-aware application. Wirel. Netw., 8(2/3):59-68, 2002.

5. Y. Ji, S. Biaz, S. Pandey, and P. Agrawal. Ariadne: a dynamic indoor signal map construction and localization system. In MobiSys 2006: Proceedings of the 4 th international conference on Mobile systems, applications and services, pages 151-164, New York, NY, USA, 2006. ACM Press.

6. A. Kotanen, M. Hännikäinen, H. Leppäkoski, and T. D. Hämäläinen. Experiments on local positioning with bluetooth. In ITCC '03: Proceedings of the International Conference on Information Technology: Computers and Communications, page 297, Washington, DC, USA, 2003. IEEE Computer Society.

7. A. Lamarca, Y. Chawathe, S. Consolvo, J. Hightower, I. Smith, J. Scott, T. Sohn, J. Howard, J. Hughes, F. Potter, J. Tabert, P. Powledge, G. Borriello, and B. Schilit. Place Lab: Device Positioning Using Radio Beacons in the Wild. 2005.

8. A. LaMarca and E. de Lara. Location Systems: An Introduction to the Technology Behind Location Awareness. Morgan \& Claypool Publishers, 2008.

9. A. Lamarca, J. Hightower, I. Smith, and S. Consolvo. Self-mapping in 802.11 location systems. In Proceedings of the Seventh International Conference on Ubiquitous Computing (Ubicomp 2005), Lecture Notes in Computer Science, pages 87-104. Springer-Verlag, 2005.

10. S. N. Patel, K. N. Truong, and G. D. Abowd. Powerline positioning: A practical sub-room-level indoor location system for domestic use. In P. Dourish and A. Friday, editors, Ubicomp, volume 4206 of Lecture Notes in Computer Science, pages 441-458. Springer, 2006.

11. N. B. Priyantha, A. Chakraborty, and H. Balakrishnan. The cricket locationsupport system. In MobiCom '00: Proceedings of the 6th annual international conference on Mobile computing and networking, pages 32-43, New York, NY, USA, 2000. ACM Press.

12. L. Reyero and G. Y. Delisle. Always best located, a pervasive positioning system. In Wireless Pervasive Computing, 2007. ISWPC '07. 2nd International Symposium on, 2007.

13. Skyhook. Skyhook Wireless, 12 June 2009. http://www.skyhookwireless.com/ howitworks/.

14. A. Varshavsky, E. de Lara, J. Hightower, A. LaMarca, and V. Otsason. Gsm indoor localization. Pervasive Mob. Comput., 3(6):698-720, 2007.

15. A. Varshavsky, A. Lamarca, J. Hightower, and E. de Lara. The skyloc floor localization system. In Pervasive Computing and Communications, 2007. PerCom '07. Fifth Annual IEEE International Conference on, pages 125-134, 2007.

16. A. Varshavsky, D. Pankratov, J. Krumm, and Eyal de Lara. Calibree: Calibrationfree localization using relative distance estimations. In Proceedings of the 6th International Conference on Pervasive Computing (Pervasive), volume 5013 of LNCS, pages 146-161. Springer, 2008.

17. F.-L. Wong and F. Stajano. Location privacy in bluetooth. In R. Molva, G. Tsudik, and D. Westhoff, editors, ESAS, volume 3813 of Lecture Notes in Computer Science, pages 176-188. Springer, 2005. 